\title{
Acquired (Non-Wilsonian) Hepato-Cerebral Degeneration in a Case of Decompensated Chronic Liver Disease- An Underdiagnosed Condition in Patients with Chronic Liver Disease
}

\author{
Pratik Saha1 ${ }^{1}$ Gautam Guha², Subhankar Chatterjee ${ }^{3}$
}

\begin{abstract}
${ }^{1}$ Resident Physician, Department of Internal Medicine, Calcutta Heart Clinic and Hospital, Kolkata, West Bengal, India. ${ }^{2}$ Consultant Neurologist, Department of Neurology, Calcutta Heart Clinic and Hospital, Kolkata, West Bengal, India. ${ }^{3}$ Guarantor, CCU in Charge, Calcutta Heart Clinic and Hospital, Kolkata, West Bengal, India.
\end{abstract}

\section{INTRODUCTION}

Most patients with DCLD have neurological problems and most of these are due to HE which responds quickly to medical measures aimed at reducing the ammonia level in blood. However, a small subset of these neurological manifestations may be attributed to a rare and understudied entity named Acquired Hepatocerebral Degeneration (AHD) which has presentation similar to Wilson's disease, ${ }^{1,2}$ but is due to manganese deposition in basal ganglia, ${ }^{3,4}$ an aetiology different from Wilson's disease and is also irreversible except for the rare incident of reversal following liver transplant, hence the nomenclature separating the two entities. The following is such a case of AHD in a 61-year-old man with cryptogenic DCLD admitted in our hospital.

\section{PRESENTATION OF CASE}

A 61-year-old man with history of chronic liver disease for 3 years, vitiligo, and hypothyroidism presented to the hospital with gradual onset speech difficulties, abnormal movements of face and mouth, involuntary tremors of hands and walking difficulties for 7 days. Patient had 2 episodes of HE before, latest being 2 weeks before current admission and had one episode of hematemesis from varices 3 months back for which endoscopic variceal ligation was done. Patient complained of weakness, fatigue, malaise, nausea, weight loss, abdominal discomfort and abdominal swelling for 1-week, easy bruising and recent-memory problems. History was negative for jaundice, pruritus, blood transfusion, family history of CLD, alcohol abuse, IVDU, high risk sexual behaviour, diabetes, hyperlipidemia, PUD, NSAID use, changes in consciousness level, inversion of sleep cycle, behavioural changes, fever.

Physical examination was positive for postural and action tremors, truncal ataxia, cog wheel rigidity, coarse dysarthria, orobuccolingual dystonia, bradykinesia, ascites, palmar erythema, muscle wasting, splenomegaly and negative for asterixis, focal neuro-deficits, Babinski sign, myoclonus, hyper-reflexia, caput medusae, spider angioma. KF rings were not present. Abdomen was soft and nontender, distended with full flanks and positive shifting dullness. Patient had very minimal drowsiness, well oriented to time place and person. MMSE was positive for short term memory, recall, concentration and negative for immediate memory, long term memory and judgement. Reitan test was positive with a time of $2 \min 20$ seconds.

\section{CLINICAL DIAGNOSIS}

Cryptogenic DCLD with possible minimal HE.
Corresponding Author:

Dr. Gautam Guha,

Consultant Neurologist,

Department of Neurology,

Calcutta Heart Clinic and Hospital,

Kolkata, West Bengal, India.

E-mail: pratik.saha829@gmail.com

DOI: $10.14260 / \mathrm{jemds} / 2019 / 624$

Financial or Other Competing Interests: None.

How to Cite This Article:

Saha P, Guha G, Chatterjee S. Acquired (nonWilsonian) hepato-cerebral degeneration in a case of decompensated chronic liver disease- an underdiagnosed condition in patients with chronic liver disease. J. Evolution Med. Dent. Sci. 2019;8(37):28732875, DOI: 10.14260/jemds/2019/624

Submission 22-07-2019,

Peer Review 30-08-2019,

Acceptance 07-09-2019,

Published 16-09-2019. 


\section{PATHOLOGICAL DISCUSSION}

USG abdomen revealed chronic liver disease with portal hypertension. UGIE revealed obliterated esophageal varices and mild PHG. Abdominal paracentesis was done which ruled out SBP. Viral serology for hepatitis markers were negative. EEG study was normal. Wilson's disease was ruled out based on age of presentation, absence of KF rings and confirmed with low serum ceruloplasmin levels $(30 \mathrm{mg} / \mathrm{dl})$. The usual causes of CLD and precipitators of hepatic encephalopathy were ruled out by history, physical examination and lab reports as mentioned.

\begin{tabular}{|c|c|c|c|c|c|c|c|c|c|}
\hline$\grave{\Delta}$ & 胥 & 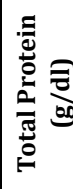 & 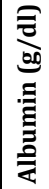 & 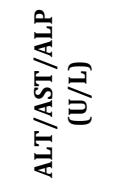 & 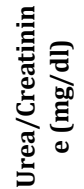 & 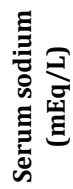 & & 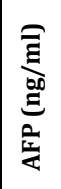 & ڤ \\
\hline D0 & $3.8 / 1.7$ & 7.1 & 2.8 & $30 / 46 / 148$ & $17 / 1.2$ & 118 & $21 / 2.08$ & 11.1 & 140 \\
\hline D4 & $3.5 / 1.5$ & 6.7 & 3.3 & $40 / 36 / 122$ & $28 / 0.99$ & 130 & $15 / 1.37$ & & 60 \\
\hline
\end{tabular}

MRI of brain was done which showed the hyper signal intensities in bilateral occipital lobe and basal ganglia, specifically the globus pallidus in T1 weighted images which is considered to be the typical finding in AHD. However, this is not pathognomonic for AHD as it may also be found in chronic liver disease of long duration. This feature may also be found in osmotic demyelination syndrome.

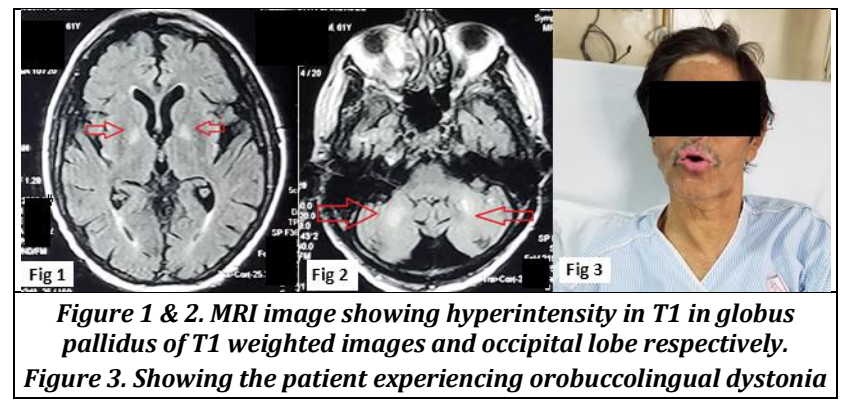

AHD is a neurological entity occurring in patients with DCLD or portosystemic shunt. The actual pathogenesis of this disease is poorly understood but is thought to be due to accumulation of manganese in certain parts of the brain, most commonly the basal ganglia. The amount of manganese deposition may correlate with the intensity of MRI signal but not with the neurological findings. The management of AHD is very challenging and controversial. Most have reported neurological improvement and complete resolution of MRI features with transplant 5,6 while some have reported no improvement. ${ }^{7}$ A case report by Luis Servin-Abad discussed a patient with AHD who underwent liver transplant 3 times and had complete resolution of symptoms and MRI features each time she underwent liver transplant but the symptoms recurred each time as the graft got damaged due to chronic rejection.

The incidence of AHD is higher than it was previously considered but the disease is lacking documentation and literature leading to lack of awareness about the disease The lack of awareness as mentioned above along with the typical initial presentation and MRI feature of AHD may confuse health care practitioners to confuse AHD with vascular diseases which might lead to unnecessary treatment adverse outcomes due to said treatments. Presence of irreversible EPS symptoms may not lead to much change in mortality but further reduce the quality of life in DCLD patients. However, with the advent of new 3D bio printing technologies ${ }^{8}$, in the future when liver transplant becomes more readily available, the inclusion of AHD in scoring systems such as MELD might be a good question that we need to keep in mind, a question only time will tell.

\section{DISCUSSION OF MANAGEMENT}

Patient was started on diuretics, beta blockers, fresh frozen plasma, antibiotics and Anti HE treatment which included Lactulose, Rifaximin.

Although autoimmune aetiology was considered due to history of hypothyroidism and vitiligo, autoimmune profile was not done due to high cost and futility of immune modulation in a case of DCLD. The patient's laboratory results including serum ammonia level improved after treatment along with improvement in patient's sensorium; however, patient's movement issues and speech issues were persisting. Thus, in the absence of Wilsons disease and persisting extrapyramidal symptoms, after neurological consultation, a diagnosis of acquired non-Wilsonian hepato-cerebral degeneration was made, the only treatment for which is liver transplant based on previous case reports. Patient had a MELD score of 21 points on admission and 15 points after 4 days of treatment and a CTP score of 9 (class B) throughout. Option of liver transplant was discussed but patient refused.

\section{FINAL DIAGNOSIS}

Acquired (Non-Wilsonian) Hepato-Cerebral Degeneration in a Case of Decompensated Chronic Liver Disease

Abbreviations- DCLD: Decompensated Liver Cirrhosis; AHD: Acquired Hepato-Cerebral Degeneration; HE: Hepatic Encephalopathy; IVDU: Intravenous Drug Abuse; KF Rings: Kayser Fleischer Rings; UGIE: Upper GI Endoscopy; PHG: Portal Hypertensive Gastropathy; SBP: Spontaneous Bacterial Peritonitis MELD: Model for End-Stage Liver Disease; CTP: Child-Turcotte-Pugh

\section{REFERENCES}

[1] Victor M, Ropper AH. Adams and Victor's Principles of Neurology. $7^{\text {th }}$ edn. New York: McGraw-Hill Publication 2001: p. 1185-96.

[2] Victor M, Adams RD, Cole M. The acquired (nonWilsonian) type of chronic hepatocerebral degeneration. Medicine 1965;44(5):345-96.

[3] Spahr L, Butterworth RF, Fontaine S, et al. Increased blood manganese in cirrhotic patients: relationship to pallidal magnetic resonance signal hyperintensity and neurological symptoms. Hepatology 1996;24(5):111620. 
[4] Krieger D, Krieger S, Jansen O, et al. Manganese and chronic hepatic encephalopathy. Lancet 1995;346(8970):270-4.

[5] Stracciari A, Guarino M, Pazzaglia P, et al. Acquired hepatocerebral degeneration: full recovery after liver transplantation. J Neurol Neurosurg Psychiatry 2001;70(1):136-7.
[6] Powell EE, Pender MP, Chalk JB, et al. Improvement in chronic hepatocerebral degeneration following liver transplantation. Gastroenterology 1990;98(4):1079-82.

[7] Counsell C, Warlow C. Failure of presumed hepatic myelopathy to improve after liver transplantation. J Neurol Neurosurg Psychiatry 1996;60(5):590. 